\title{
Particle-Wave Duality Resulting from the Granulation of Fields in a Hypercubic Lattice
}

\author{
Christiaan T. de Groot \\ University of Applied Sciences Utrecht, Utrecht, Netherlands \\ Email: ctdegroot@live.nl
}

How to cite this paper: de Groot, C.T. (2021) Particle-Wave Duality Resulting from the Granulation of Fields in a Hypercubic Lattice. Journal of Modern Physics, 12, 870886.

https://doi.org/10.4236/jmp.2021.127055

Received: April 7, 2021

Accepted: May 18, 2021

Published: May 21, 2021

Copyright (อ 2021 by author(s) and Scientific Research Publishing Inc. This work is licensed under the Creative Commons Attribution International License (CC BY 4.0).

http://creativecommons.org/licenses/by/4.0/

(c) (i) Open Access

\begin{abstract}
The possibility of granulated discrete fields is considered in which there are at least three distinct base granules. Because of the limited size of the granules, the motion of an endlessly extended particle field must to be split into an inner and an outer part. The inner part moves gradually in a point particle-like fashion, the outer is moving step-wise in a wave-like manner. This dual behaviour is reminiscent of the particle-wave duality. Field granulation can be caused by deviations of the structure of the lattice at the boundaries of the granule, causing some axes of the granule to be tilted. The granules exhibit relativistic effects, inter alia, caused by the universality of the coordination number of the lattice.
\end{abstract}

\section{Keywords}

Discrete Space, Granular Fields, Hypercubic Lattice, Motion in a Lattice, Particle-Wave Duality, Relativistic Effects

\section{Introduction}

A duality in the movement of particles in a discrete space can only be established if the possibility of motion in a lattice can be described. The problem of motion in a discrete space has a long history. It is first formulated by the Arrow Paradox of the ancient Greek philosopher Zeno of Elea. According to the interpretation of Grünbaum [1], the Arrow paradox states that it would be impossible to perceive any difference between a moving and a motionless object at the smallest moment of a discrete space-time. More recently, B. Russell [2] also highlighted the problems with the movement of point-shaped particles in a lattice. When the object is a field, the entities in space determining it are coupled to the vertices of the lattice, then when the field moves, the vertices must also move. Because of the time immobility of the vertices of a lattice, Čapek Milič and others have seen this as an impossibility [3]. 
Here too, the immobility of the vertices is a basality, but then in a four-dimensional lattice. It is shown that three-dimensional areas within a four-dimensional discrete space can be in motion relative to the surrounding lattice when the areas have boundaries that provide for the transition of the moving inner region and the still outer space. When motion in a discrete space is restricted to bounded areas, it is obvious to investigate if fields in a discrete space can also be composed of bounded areas. The second purpose of the article is therefore to get an idea of the properties of the most common fields consisting of granules, i.e. bounded three-dimensional areas in a four-dimensional discrete space. For this purpose, the so-called discrete field needs only be composed of three different types of basic granules, including a moving granule, done in Chapter 2.

Chapter 3 deals with the properties the divers basic granules must have in order the granulated fields correspond as closely as possible to reality. Considering fields as an ensemble of granularities of limited dimensions opens up new explanation opportunities, especially when describing the motion of an endlessly expanded particle field. In that case, two modes of motion are needed consistent with the de Broglie's hypothesis when the granular fields are part of a multidirectional hypercubic lattice, as shown in Chapter 4.

The purpose of how granule movement in a lattice can take place, is explained in Chapter 5, showing that the granularities may originate from aberrations of the structure of a hypercubic lattice in the boundaries. To show how the movement of a granule looks like, a Minkowski diagram-like visualisation has been applied to a lattice. It is also shown that in a lattice, under certain conditions, relativistic effects are present within the moving granule.

\section{The Granulation of Fields in a Lattice-Like Discrete Space}

In a hypercubic lattice, all vertices in time and space are connected by eight edges (coordination number). If this four-dimensional lattice is taken as a frame of reference, where the fields are somehow connected to the vertices, all the phenomena from the past and the future are also linked. Consequently, there are two points of view to look at phenomena in the form of fields, both of which are used in this article:

- One is the four-dimensional perspective within which a phenomenon is the time development of an unchanging four-dimensional presence extending from the past into the future. Also called eternalism or block-universe [4].

- The other is the spatial perspective in which a phenomenon is a concatenation of a whether or not in time changing three-dimensional presence.

\subsection{The Discrete Field}

In a continuous space, a field is defined as a quantity present at any location in space. In a lattice as discrete space, the location consists of vertices where each vertex has a limited number of relations with other vertices. A field in a lattice needs to be expressed as a set of quantities related to the vertices. The field thus discretized limits the possibilities in the description of a field. 
In a hypercubic lattice all vertices have the same coordination number. Assuming this also holds when fields are present, it means that there is a large number of vertices with edges having field characteristics in addition to the function of position determination in space. So there are field vertices of which at least two of the eight edges are needed to determine the position within the space and the rest can be field edges. Then, to allow for a diversity of field strengths, massive concentrations of field vertices are needed. Because large field strength must occur everywhere in space, the space would only consist of field vertices. If so, the vertices lose their function as the smallest entities of a fourthdimensional space-time. This can be solved by a much larger coordination number or by positioning the field vertices in the boundaries of granules.

\subsection{The Granular Character of the Discrete Field}

Another possibility is that interaction stems from the coincidence over time of three-dimensional regions with field action. Chapter 5 outlines how such an area could look like. The discrete field is then a coherent set of three-dimensional areas of various sizes $L$, expressed in the unit distance $\Delta x$ of a lattice.

From a three-dimensional perspective, the three-dimensional area is a flat spatial plane that extends over time, called the active plane. A variety of field strengths is possible when the discrete field consists of a series of active planes at regular distance $L$. The spatial density of the field is then $1 / L$. The probability of two active planes of different fields with the same action coincide is $1 / L^{2}$.

With the active planes of size $L^{2}$ at mutual spatial distances $L$, the discrete field can be split into granules of size $L^{3}$ having the action at the boundaries of the granule. This allows the discrete field to be divided into separate non-overlapping granules of size $L^{3}$.

\subsection{The Field Granule from Discretization of the Units}

The likelihood of a granulated field also arises from the transformation of the usual units into the two units $\Delta x$ and $S$ of a hypercubic lattice with multiple directions [5], where $\Delta x$ is the smallest distance of a lattice and $S$ stands for the number of lattices in which a phenomenon is present. The conversion results in an expression of the field strength in one lattice or subspace:

$$
E_{\text {sub }}=1 / L_{d}^{2} \quad 1 / \Delta t
$$

in which $L_{d}$ is a quantity analogue to the discrete distance between two particles.

The inverse proportionality with an area indicates the existence of a field granule, or field granule of spatial size $L_{d}^{3}$. The conversion also results in a smallest field granule for the electron (electron radius) has the enormous local magnitude of about $L_{d 0}=3.38 \times 10^{19} \Delta x$, expressed in smallest lattice distance.

\section{The Discrete Field in One Subspace Described with Field Granules}

Suppose that the granules of the discrete field have the shape of a four-dimen- 
sional cube, with the dimension $L^{3}$ in space and an extension $L$ in time, where the cause of the field effects is present in the spatial boundaries. Chapter 5 examines the possibility that the field effect is caused by aberrations of the structure of the four-dimensional hypercubic lattice.

It turns out that these active boundaries are repeated after the time $T_{\text {field }}$ and are interconnected by the corner vertices of the cube. The corner vertices form the basis of interconnectedness of granules allowing the various types of granules to form extensive fields.

\subsection{The Three Different Base Field Granules in Each Subspace}

Three different types of field granules are required to describe the most common phenomena.

\section{Still field granule;}

The still field granule is immobile relative to the local discrete space. The field granule is regularly repeated every $T_{\text {field. }}$. There can be different types of still field granules, each representing another type of action like electric or magnetic. It is assumed that the cause of the action is present in one of the side planes of the cubic field granule, making the presence frequency of an active plane $1 / T_{\text {field. }}$. An example of a such plane with local deviation of the axis declination is described in Section 5.2. The inner space of the field granule is void, that is, a regular lattice.

\section{Moving granule;}

The moving granule, called moving space, is a cube with four moving side planes that ensures the regular interior space to move with relativistic effects. Section 5.5 describes the moving space in a lattice. This possibility is visually illustrated in Figure 1. The moving plane is a three-dimensional bounded area in $\mathrm{R}^{4}$, whose entire inner space is moving with speed $v$ and the boundaries are such it forms the transition between the still outer space and the moving inner space. A more precise description of such a boundary is shown in Section 5.3 in the case of a lattice. The relativistic effects are showed in Section 5.4.

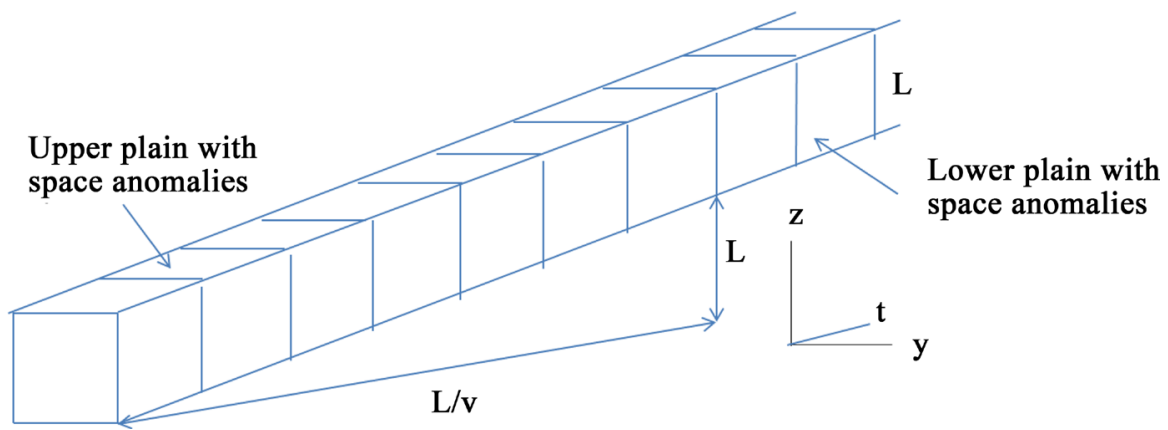

Figure 1. The moving granule. In the moving space, the entire space between top and bottom planes moves integrally with respect to the space around the granule caused by the anomalies in the space structure in the upper and bottom planes. The anomalies caused that every $t=1 / L$ the space to be changed from a still to a moving space at the top and vice versa on the bottom plain. 


\section{Jumping field granule moving at maximum speed;}

A repeating space jump in time is another mode of movement of a field granule in a lattice. The field granule is present at one location during the time $T$ before jumping to a successive location $L(=T)$ in the direction of motion. In $\mathrm{R}^{4}$, the sequence of jumping field granules is located along the space-time diagonal. Seen from $\mathrm{R}^{3}$, the presence of the field granule moves with discrete speed $v_{d}=L / T=1$. In Figure 2, the four-dimensional situation is roughly outlined, with the jumping field granule depicted as a three-dimensional cube.

To be in accordance with reality, it is supposed that the side faces of the cube to consist of two orthogonal planes with a $E$ and $B$ action.

The granular field is an ordered combination of various types of field granules forming constructions regularly distributed in space and time and interconnected via the corners. Next, it is investigated what the properties of granular fields will be if they are described as a combination of said granules.

\subsection{The Movement of a Homogeneous Electric and Magnetic Granular Field}

Take the constructive combination of still and jumping field granules. The still $E$ (or $B$ ) field is a time chain of still field granules. Let the moving field be a time chain of still $E$ (or $B$ ) and jumping $E, B$ field granules of the same size $L$, interconnected at the corner vertices, replacing one of a still field granules in the time

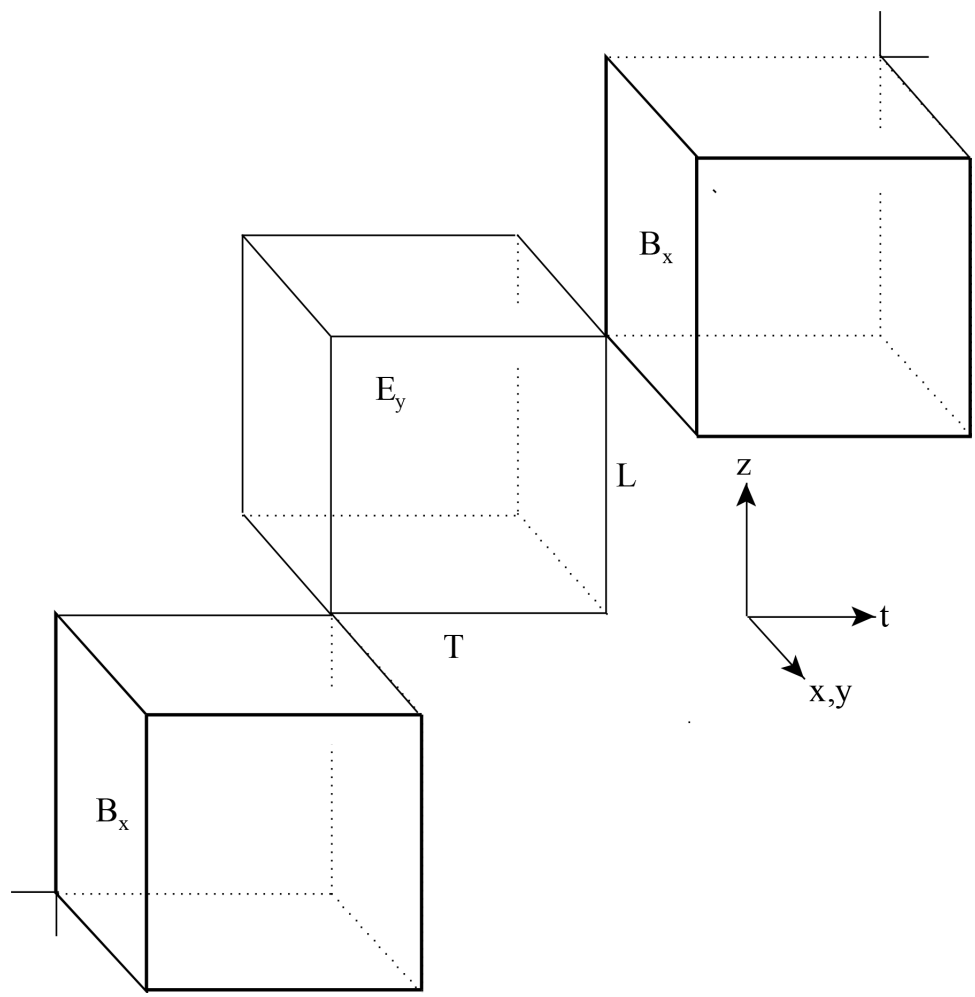

Figure 2. A jumping field granule. The jump-like motion of field granules is depicted in $\mathrm{R}^{4}$ as a series of cubes. The cubes are interconnected sequentially in the corner vertices, such that the cubes are present along the diagonal of space-time. 
chain by a jumping field granule at each $T_{\text {jump }}=L / v$. So, the time chain of field granules makes each $T_{\text {jump }}$ a jump Lin the direction of movement. Because the jumping field granule consists of both $E$ and $B$ fields as presumed, the $E$ field is present at all times. The jumping field granule is the cause of the presence of the $B$ field during the short period $T(=L)$ every $T_{\text {jump. }}$.

It is obvious that the field strength is proportional to the time the field is present to perform its operation, i.e. has the probability of coinciding with another $E$ or $B$ field granule. Then the fraction $B / E$ is equal to the ratio of the time periods in which the fields are present, so that $B / E=T / T_{\text {jump }}=v$ :

$$
B=v E,
$$

or when $B$ is the main field: $E=v B$.

Equation (1) is in accordance with the empirical expression of a moving electric or magnetic field, showing that the discrete field can be expressed in field granules.

The disadvantage of het above model is that it misses the reason for the repetition time $T_{\text {jump }}$, making the model incomplete.

\subsection{The Motion of a Granular Particle Field}

Due to the spatial in homogeneity of the particle field, the motion of a granular particle field must be split into two of the above types of motion acting simultaneously. Described in granules, an endlessly extending particle field will consist of a series of concentric field granules of increasing size. A moving granulated particle field necessarily consists of the equal speed of all individual granules of different sizes. This makes that none of the three basic granules alone can describe the motion of the discrete particle field, the solution is a combined presence of a moving space (granule) and jumping granules. To this end, the motion of the particle field is split into an inner and outer sphere, the inner part is being moved by one moving granule with rib $L_{\text {moving. }}$. The outer part by a series of combined still and jumping granules of suitable size moving in the manner of a homogeneous field as described in the previous paragraph.

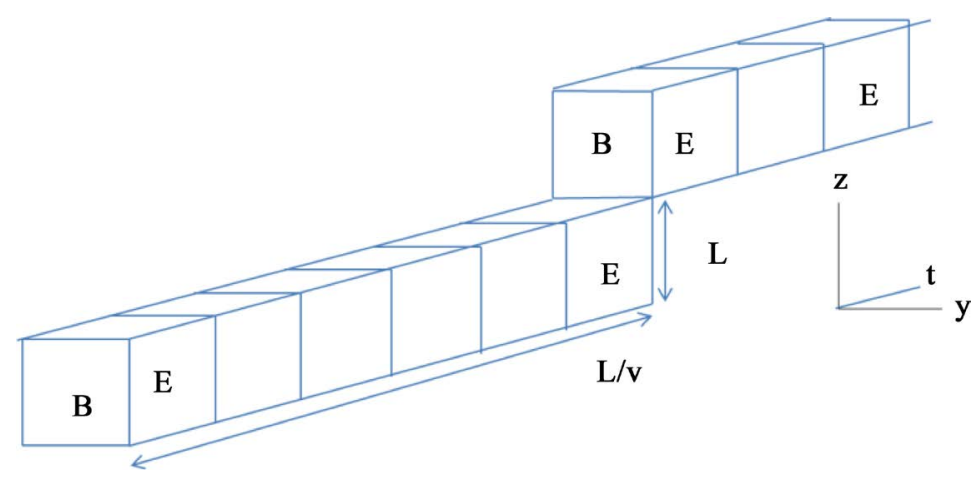

Figure 3. A moving homogeneous field. A moving homogeneous field is a combination of a still and a jumping field, depicted in $\mathrm{R}^{4}$ as a series of cubes. During the time $t=L / \mathrm{V}$ the field stands still, then the jumping granule causes a jump over the distance $L$. The cubes are interconnected forming a constructive unity. 
The smallest part of the particle is present as a locally still field within the moving space and will, as part of the moving space, move gradually with discrete velocity $v_{d}=1 / L_{\text {moving }}$ relative to the surrounding space. Because the inner part is part of the moving space, it also has time dilation and length contraction.

Using the visualisations of Figure 1 and Figure 3, the moving granule of Figure 1 is implanted into the granule of the moving field of Figure 3. Many concentric granules a la Figure 3 of increasing size form the outer shell of the moving particle field.

\subsection{The Momentum of the Inner Field}

The concentric field granules of the particle field have increasing discrete sizes $L_{d i}$ and the associated discrete repeat distances in time $T_{d i}$. Let $R_{\text {particle }}$ be the averaged effective spatial size of the many granules and $T_{\text {particle }}$ the mean repeat distance of the granules, determined by:

$$
1 / R_{\text {particle }}=\sum 1 / L_{d i} \quad 1 / \Delta x,
$$

both provided with the corresponding discrete units. Remarkably, these two quantities have a significant difference in size, as can be seen from ref. [6]:

$$
T_{\text {particle }}=(2 \pi / \alpha) R_{\text {particle }} \text {. }
$$

Wherein $\alpha$ is the fine structure constant. This means that in one subspace, the cube-shaped granules are only sporadically present over time. In the time in between, the field granules a represent in other subspaces. Call the particle mass in one subspace the average density of the still field granules at a given location, represented by:

$$
m_{\text {subspace }}=1 / T_{\text {particle }} \quad 1 / \Delta t .
$$

This is conform the particle mass in one subspace of reference [6].

Take a moving particle field. All particle granules with size $L_{d i} \leq L_{\text {moving }}$ are present within the moving space. It is reasonable that $1 / T_{\text {particle }}$ is mainly determined by the field granules smaller than $L_{\text {moving. }}$. Relative to the surroundings of the moving space, the density of the particle granules at successive positions in the surrounding space is given by multiplying $1 / T_{\text {particle }}$ by the discrete velocity. This will be called the momentum per subspace:

$$
p_{\text {subspace }}=v_{d} / T_{\text {particle }} \quad 1 / \Delta t \text {. }
$$

In a lattice is $\Delta x=\Delta t$ making $v_{d}$ dimensionless.

\subsection{The Momentum of the Outer Particle Field}

Each $T$ ( $=L_{\text {moving }}$ ), the moving space makes a unit-step in the direction of motion so that after $t=T^{2}$ the total size $L_{\text {moving }}$ of the cube has been covered. The outer part of the moving particle field must move in conjunction with the inner part, i.e. making a minimum jump $L_{\text {moving }}$ every $t=T^{2}$ or a multiple thereof. This is possible if the outer particle field consists of a series of concentric shells of increasing discrete size $L_{d i}$ with $L_{d 1}=L_{\text {moving }}$. To be in motion, each shell consists 
of several still field granules and one jumping field granule, which makes every discrete $t_{d i}=L_{d i} / v_{d}$ a jump $L_{d i}$ in the direction of motion.

Implicitly, every jump $L_{d i}$ fits exactly into $L_{d i+1}$, making $L_{d i}$ a plural of $L_{d 1}$. This means that, seen in $\mathrm{R}^{3}$, the spatial distribution of the outer particle field is spaced by a multiple of $L_{\text {moving. }}$. This means that during $T^{2}$ there is a still outer field, after which the still field is present at the next spatial location. Therefore there is a greater chance of interaction at mutual distances $L_{\text {moving, }}$ which can be interpreted as the wavelength $\lambda_{d}$. By doing that, the relation between the discrete wavelength $\lambda_{d}$ of a moving particle in a discrete space and the discrete velocity becomes $v_{d}=1 / \lambda_{d}$. Thus the number of outer field granules entering a new area, or the momentumof the particle field, is determined by

$$
p_{\text {subspace }}=1 / L_{\text {moving }}=1 / \lambda_{d} \quad 1 / \Delta x \text {. }
$$

This relation applies next to relation (5).

In one subspace, the wave length $\lambda_{d}$ is expressed in units of one lattice. In the next chapter, relations (5) and (6) will be expanded to a space with multiple lattices. The interpretation $\lambda_{d}=L_{\text {moving }}$ links the wavelength to the particle mass via Equation (9).

\subsection{Variety of Speeds of the Particle Field}

The jumping outer part of the moving particle resembles the jerking motion described in [7] [8] or [9]. The description of a moving particle given here is not only more extensive than in these references, it also enables a wide variety of velocities. To clarify this, take the situation where the moving particle consists of a series of concentric moving granules. Then, going from the outside in, there is an increase of velocity and time dilation caused by the successive moving spaces. According to the relativistic relations, the effect of one of the moving granules sized $L_{i}$ on velocity and time dilation is determined by $T_{i+1}^{2}=T_{i}^{2}\left(1-v_{i}^{2}\right)$ with $v_{i}=1 / L_{i}$. With $k$ moving spaces, the cumulative effect is $T_{k+1}^{2}=T^{2} \Pi\left(1-v_{i}^{2}\right) \mid i=1, \cdots, k$. This makes that the velocity $v_{t o t}$ of the inner moving granule is determined by: $T^{2}\left(1-v_{\text {tot }}^{2}\right)=T^{2} \Pi\left(1-v_{i}^{2}\right) \mid i=1, \cdots, k$, making

$$
v_{\text {tot }}^{2}=1-\Pi\left(1-1 / L_{i}^{2}\right) \approx \sum v_{i}^{2}-\cdots
$$

With this expression any velocity can be obtained by a suitable combination of $L_{i}$.

\section{Granulated Fields in a Multidirectional Discrete Space}

In a hypercubic lattice, the movement of the particle field can only take place along one of the three spatial axis, which is very unrealistic. In a more realistic multidirectional discrete space, the entire space is split up into $n_{\text {directions }}$ subspaces each with their own set of spatial directions [5]. The number of spatial directions $n_{\text {directions }}$ comprises at least $10^{22}$ subspaces. The set of subspaces $n_{\text {directions }}$ is a small subset of all subspaces $n_{\text {subspaces }} ; n_{\text {subspaces }} \gg n_{\text {directions }}$, allowing the particle field $n_{\text {particle }}$ to be in a multiple of $n_{\text {directions. }}$. 


\subsection{Movement of a Particle Field in Multiple Subspaces}

When applying the mode of motion of a moving spherical symmetrical particle field from the previous chapter in a multidirectional space, the field is present in at least $n_{\text {directions }}$ subspaces. Each subspace, if necessary, provided with a subspace with an axis in the direction of motion. The necessity for the outermost regions of the moving particle field to have a subspace with an axis in the direction of movement ensures that the number of these subspaces is also $n_{\text {directions. }}$. Consequently, the moving particle field is present in $n_{\text {directions }}$ subspaces with an axis in the direction of motion next to the $n_{\text {directions }}$ subspaces with random axes. The different subspaces are interconnected via the space-point. The space-point of a multidirectional lattice is a time series of vertices connected in time, the vertices being part of subspaces with different spatial directions ref. [5].

\subsection{The Momentum of a Particle Field in a Multidirectional Lattice}

In section 3.4 it has been determined that per subspace the densityof a field granule of the particle is $m_{\text {subspace }}=1 / T_{\text {particle }}$. Multiplication of this particle mass per subspace by the number of subspaces $n_{\text {particle }}$ gives the total densityof the particle field expressed in the two units of a multidirectional discrete space:

$$
m_{\text {discrete }}=n_{\text {particle }} / T_{\text {particle }} \quad S / \Delta t \text {. }
$$

In reference [6] it has been shown that only the discrete units $\Delta x$ and $S$ are needed to express the physical quantities in a multidirectional lattice. Also in reference [6], the same mass expression (7) has been found.

With (5), the density of the particle granules in consecutive positions with respect to the particle's surrounding space is

$$
p_{\text {discrete }}=v_{d} n_{\text {particle }} / T_{\text {particle }} \quad S / \Delta t \text {. }
$$

By inserting the mass expression (7) herein, the momentum $p_{d \text { particle }}$ expressed in discrete units is

$$
p_{d \text { particle }}=m_{\text {discrete }} v_{d} \quad S / \Delta t,
$$

also in accordance with reference [6].

\subsection{The Movement of the Outer Field Granules}

As explained in section 4.1, the outermost field of the moving particle is presented as a series of jump field grains in $n_{\text {directions }}$ subspaces that all having an axis in the direction of motion. Equation (6) describes the momentum in one subspace, i.e. the frequency with which the outer field granules in one subspace enter new areas of space. For $n_{\text {directions }}$ subspaces, the frequency is augmented by that number by which the momentum in the multi directional lattice becomes

$$
p_{d \text { particle }}=n_{\text {directions }} / \lambda_{d} \quad S / \Delta x,
$$

$\lambda_{d}$ is the discrete wavelength in the subspaces involved. Equation (9) links the wavelength to the particle mass.

In ref. [6] it is shown that the de Broglie relation in a multidirectional lattice is 


$$
p_{d}=h_{d} / \lambda_{d} \quad S / \Delta x
$$

where in $h_{d}$ is the discrete Planck's constant,being the Planck's constant expressed in the discrete units. With (9), the discrete Planck's constant becomes:

$$
h_{d}=n_{\text {directions }} \quad S \text {. }
$$

Relation (11) has also been found in reference [6] by using the same reasoning as in section 3.6 to obtain the wavelength of the granulated photon field.

\subsection{Relativistic Mass Increase of Moving Granulated Fields}

In case the number of edges per vertex is the same for every vertex of a hypercubic lattice, it is shown in section 5.4 that length contraction and time dilation (14) is present within the moving space, which is also true for the largest granule $L_{\text {moving }}$ of the moving space. Because according to Equation (6) $\lambda_{d}=L_{\text {moving }}$, the wave length is subject to length contraction

$$
\lambda_{d}^{\prime}=\lambda_{d 0} \sqrt{1-v_{d}^{2}} \quad \Delta x .
$$

Using the equation (10), the relativistic momentum is

$$
p_{d}^{\prime}=h_{d} / \lambda_{d}^{\prime}
$$

With $p=m \cdot v$ and considering that the $v$ is the velocity in the surrounding space, makes the particle mass relativistic

$$
m_{d}^{\prime}=m_{d 0} / \sqrt{1-v_{d}^{2}} \quad S / \Delta x \text {. }
$$

From the length contraction of the wave length follows the increase of the particle mass.Based on this, the following observations can be made.

According to Section 3.4, each granule of the outer paticle field $L_{d i}$ is a plural of $L_{\text {moving. }}$ When the size of $L_{\text {moving }}$ changes, the larger granules follow immediately. Apparently the successive particle granules are spatially interconnected.

Secondly, the densification by length contraction increases the presence of the outer field-units and therewith the likelihood of an encounter with another field (interaction). This is in line with the interpretation of the momentum of a granular field as the density of the particle granules at successive positions in space.

\subsection{The Dual Description of the Particle Momentum in a Multidirectional Lattice}

In a multidirectional discrete space, the endlessly extended particle field is present as a series of concentric granules of different sizes in each of the $n_{\text {directions }}$ $\left(>10^{22}\right)$ subspaces. To describe motion of an endlessly extended particle field with granules of limited sizes, it will consist of inner and outer granules. The inner field granules show a gradual displacement, represented mathematically by equation (8). The outer particle field shows step wise displacement represented by (9).

Because the largest granule within the moving space and the smallest granule of the outer part are somehow coupled, Equations (8) and (9) act simultane- 
ously. This means that that in the case of granulated fields, the momentum of a particle is mathematically described in two ways, resembling the particle-wave duality.

\section{Field Granules Arising from Anomalies in the Structure of the Lattice}

This chapter examines whether the granulation of fields can be the result of deviations in the structure of a lattice. A lattice consists of vertices and edges with a certain coordination number ( 8 for a hypercubic lattice). The structure of such a lattice is the organisation of the vertices and edges. The space structure of a hypercubic lattice with four orthogonal axes in every vertex is very simple and regular in every vertex, it is called the regular lattice.

A multitude of local structural deviations from the regular lattice are possible. Some of these will be given here whose pattern in deviations is repeated regularly along the time axis. The examples, necessarily at the level of vertices and edges, are presented visually for quick understanding.

\subsection{An Example of a Simple Anomaly in the Structure of the Lattice}

To become familiar with structural deviations, an example of a very simple anomaly in the structure of a regular lattice is given in Figure 4(a).

Generally, a structural deviation in one vertex necessarily implies that the two adjacent vertices also have a structural deviation. A series of adjacent deviating

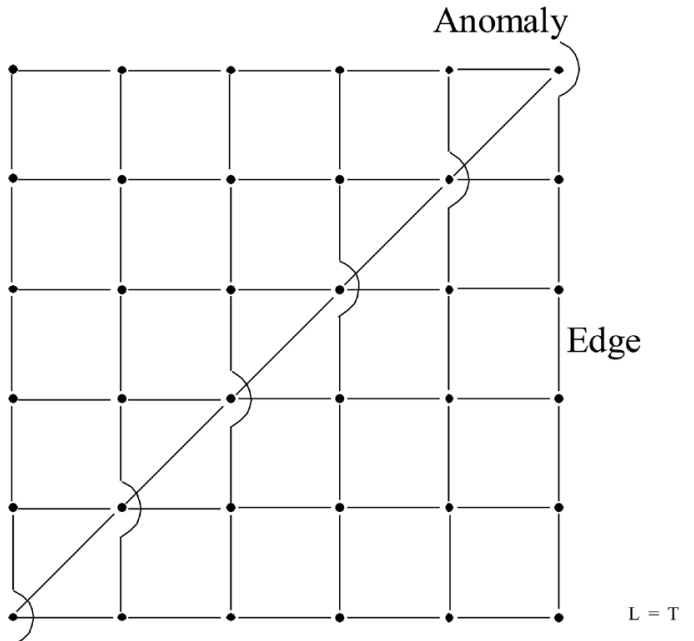

(a)

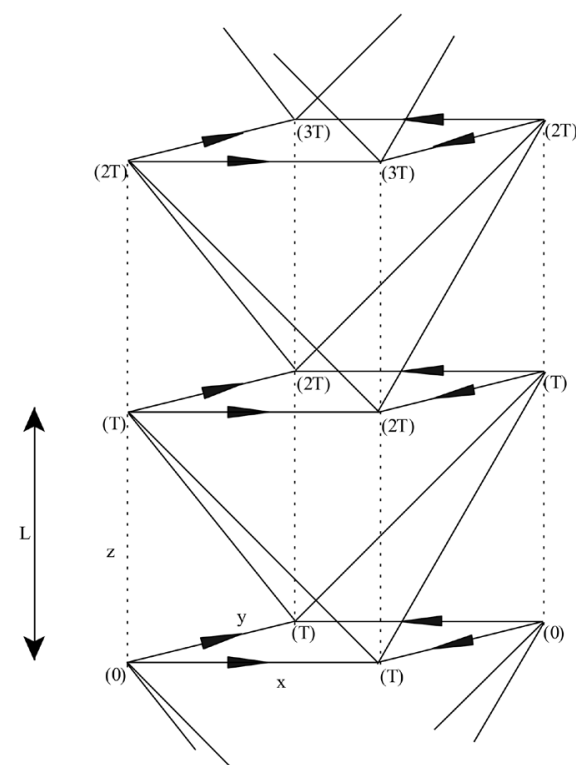

(b)

Figure 4. Constructions of anomalies within one hypercubic lattice. The left (a) shows a simple anomaly in a two-dimensional lattice forming a straight diagonal anomaly-line. (b) shows a construction of anomaly-lines in a four-dimensional lattice. The anomaly-lines diagonally in space and time are indicated by an arrow. The figure shows that a cube-like pattern of anomaly-lines of rib $L$ is present at regular intervals in time and space: $(t=0, z=0),(t=T, z=L)$, etc. Spatially seen, the pattern moves in the $z$-direction with speed 1 . 
vertices constitutes a so-called anomaly-line. Note that an anomaly-line as in Figure 4(a) locally runs diagonally between two axes, and secondly that it has no beginning or end.

In $\mathrm{R}^{4}$, constructions of anomaly-lines can exist when several lines are combined to endlessly extending repetitive structures. Figure 4 (b) illustrates such a construction. Herein four anomaly-lines merge at regular distances in space-time into one vertex. The sketched image is diagonal in space and time and is present in $\mathrm{R}^{4}$ as a static column-like construction in four dimensions.

In the construction a recurring pattern can be recognized consisting of a cubeshaped assembly of eight anomaly-lines. Each cube consists of four pure spatial anomaly-lines and four lines diagonally in space-time. By focussing on the progression in the time of the cube-shaped pattern, the pattern makes each $T$ a step $L$ along the $\mathrm{z}$-axis. The series of positions of the corner vertices of the cube can be expressed by the set $\{z, t\}=\{(i Z, i T) \mid i$ : integer, $Z=T=L\}$.

Seen in $\mathrm{R}^{3}$ the cube moves with velocity $v_{d}=i Z / i T=1$.

The assembly of anomaly lines can also be arranged so that the formed construction consists of a series of cube-like patterns extending endlessly in time. Seen from $\mathrm{R}^{3}$, these cubes stand still at one location.

\subsection{Stand-Still Three-Dimensional Regions with Tilted Axis Caused by Anomalies}

With the possibility of the last section, no physics can be present. Namely, at two intersecting moving columns there is no conceivable transition in two fully different columns.

To provide for possible interactions, consider structural deviations whose anomalies are present on the boundaries of a three-dimensional region of $\mathrm{R}^{4}$. These anomalies affect the entire inner space-time region by tilting some axes of the region. These so-called tilted axes are not parallel to similar axes of the lattice surrounding the region. Because the organization of the internal axes is locally the same as in a hypercubic lattice, the space is regular within the said region.

Due to the tilting of the axes within an entire area, there is a chance that regions overlap cannot always occur, i.e. it has an interaction as result. These three-dimensional regions in $\mathrm{R}^{4}$ form an active plane within $\mathrm{R}^{3}$.

An active plane forms an endless corridor in time with, at the top and bottom of the corridor, the anomalies that cause the axes to tilt. Figure 5 shows an example of an active plane in the form of a time slice of the three-dimensional corridor.

The cohesion of slices along the $y$-axis is enhanced when a $z$-line is missing in the corridor at every $T^{2}$, caused by two open edges, i.e. an edge with a missing vertex, at the top and bottom. A missing $z$-axis means that there is a gap in time in the active plane. Then the organization of the anomalies along the $y$-axis in the top and bottom of the corridor can be such (for example in the manner of Appendix A) that every $T(=L)$ the active plane has an open edge at the corner vertices. 


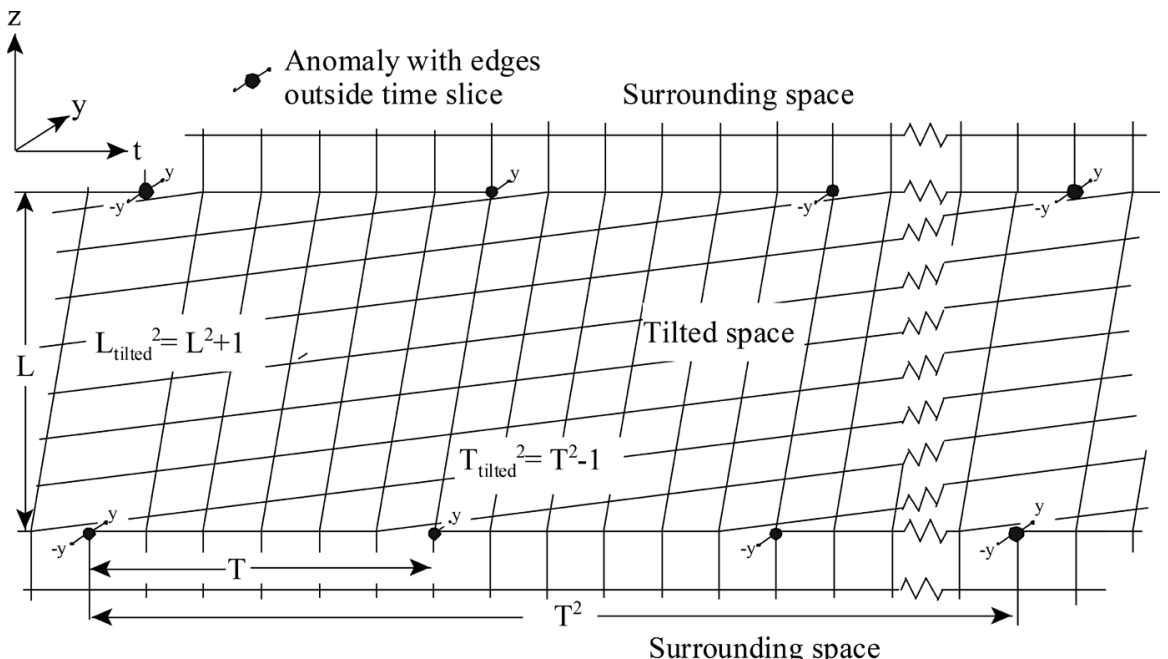

Figure 5. Time slice with tilted axes of a corridor of a still active plane. The time crosssection of the corridor of height $L$ forms a slice along the $z$-and $t$-axis of thickness $\Delta y$ $(=1)$. The lines depicted are the connecting edges, the vertices are located at the intersections of the lines. At regular intervals $T$, the anomalies at the top and bottom lack a connecting edge causing the $z$ - and $t$-axes to be relatively tilted. The missing edge on each $T$, drawn as an open, unconnected edge in the $y$-direction, is thought to be connected to a vertex of the adjacent slice.

Based on Figure 5, two distinct active planes can be distinguished, one with tilted $z$ - and $t$-axes and the other with tilted $y$-axis and $t$-axis. This distinction makes that, apart from opposite spatial tilt direction, the active plane can have six different spatial orientations in $\mathrm{R}^{3}$.This is reminiscent of the six different $E, B$ operating axes.

Active planes of opposite tilt cannot merge, which means that the merging of active planes is not possible in all cases. The said unconnected open edge at the corners each $T$ allows to arrange several active planes into a cube, with which various field granulesof Section 3.1 can be formed.

\subsection{Anomalies Causing the Movement of a Three-Dimensional Regions}

By taking a small variation in the structure of Figure 5, an entire spatial plane is in motion. Figure 6 shows the structure of such plane, in which the time axis makes a spatial step $\Delta z$ in the direction of motion each $T$. Herein, the anomalies at top and bottom are such that all time lines of the corridor and boundaries are relatively inclined in the direction of motion, where the tilted time axes having no beginning or end.

The time slice of the moving plane consists of the repeated occurrence of $T$ segments in which one of the segments makes a jump in time due to a missing $Z$-axis. The moving plane is formed by a series of $L(=T)$ adjacent time slices in such a way that segments with missing $z$-axis are never present at the same time.

Seen in $\mathrm{R}^{4}$, the corridor with the moving plane looks static. In $\mathrm{R}^{3}$, the plane is in consecutive positions along the $z$-axis over time, which means it is in motion. 


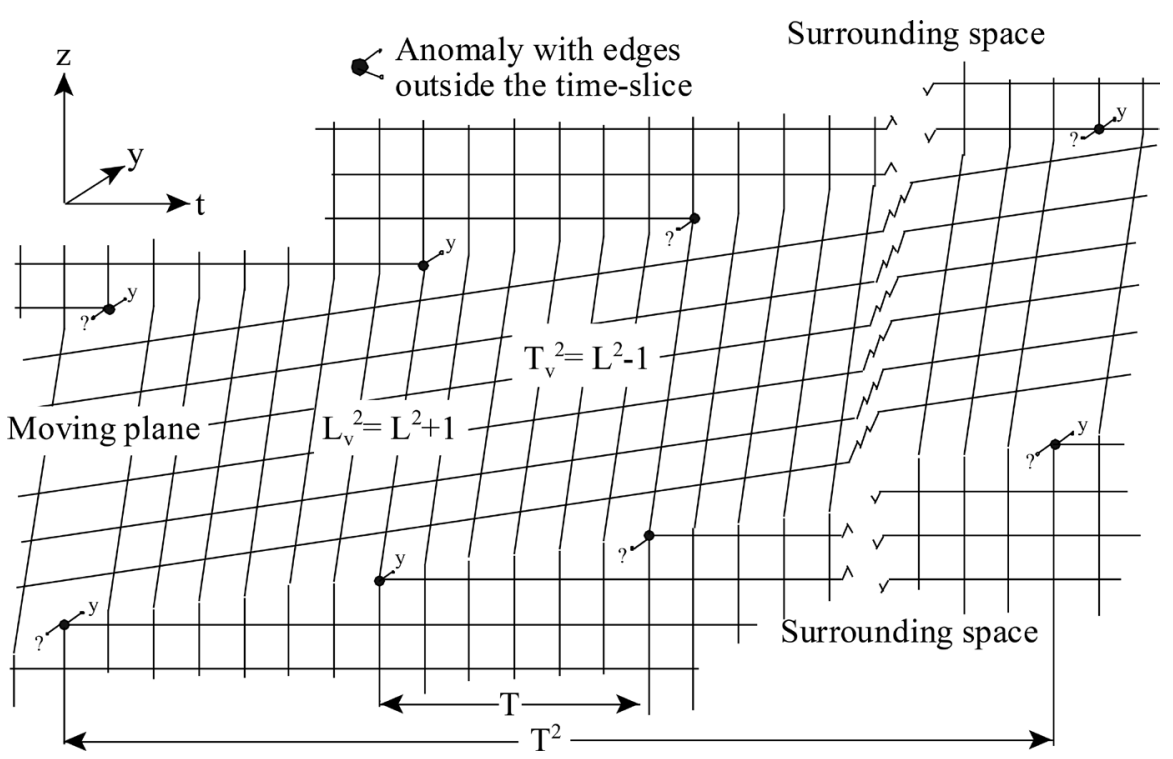

Figure 6. A time slice of a moving plane. Depicted the edges of a time slice of a moving plane with the vertices at the intersections. In contrast to the tilted plane, the inner vertices are shifted by the unit-distance on each $T(=L)$. This organization results in two or four unconnected edges each $T$, which are connected with adjacent slices. In a multidirectional lattice, the tilted time lines should be read as the (moving) space-points.

As can be seen in Figure 6, all axes with in the corridor are tilted in the same way so that the entire space of the corridor has a locally regular space structure that moves at local speed $v_{d}=1 / T$.

In the case of a moving space-point the set of subspaces must be such that one of them has an axis in the direction of motion. This subspace sets the space-point in motion.

\subsection{Length Contraction and Time Dilation within a Moving Plane}

An obvious definition for the distance between two vertices on an axis is the number of intermediate edges. Using this definition, the time distance within the moving plane is shorter than the comparable time distance in the surrounding space because of the missing $z$-axis in the moving plane. The time distance $T^{2}$ in surrounding space has a corresponding time interval $T_{v}^{2}$ within the moving plane, which, due to the time gap, is one unit-distance less: $T_{v}^{2}=T^{2}-1$. Seen from the surrounding space, combined with $v=1 / T$, the time distance $T_{V}$ within the moving plane becomes: $T_{v}=T \sqrt{1-v^{2}}$.

As Figure 6 shows, the time axis within the moving plane is literally stretched relative to the time axis of the surroundings. Take the time distance $\Delta t$ within the moving plane. In surrounding space, this time interval lasts longer, i.e. it comprises a larger number of $\Delta t^{\prime}$ vertices met: $\Delta t^{\prime} / \Delta t=T / T_{v}$. Using the above expression for $T_{b}$ the relation between the time intervals $\Delta t^{\prime}$ and $\Delta t$ becomes

$$
\Delta t^{\prime}=\Delta t / \sqrt{1-v^{2}}
$$

being the time dilation of events. 
In the corridor the smaller number of vertices overtime is compensated by an increase in vertices in the direction of movement. To clarify this: The corridor extending indefinitely in time has, in addition to the boundary in upper and lower surfaces, also two side surfaces as boundaries. At the location of the side surfaces, the inner vertices are connected to the vertices outside of the corridor.

Take the side surface $L . T$ expressed in distances of the surrounding space. The same side surface, expressed in distances of the corridor, comprises of $L_{V} T_{V}$ vertices, where $L_{V}$ is the mean number of vertices forming the height. At the surfaces, each vertex of the corridor is connected with a vertex of surrounding space, making $L_{v} T_{v}=L T$. As a result, the average number of vertices within the moving plane in the direction of the velocity is

$$
\Delta z^{\prime}=\Delta z \sqrt{1-v^{2}} .
$$

Take a number of vertices $\Delta z$ within the moving plane in the direction of motion. Seen from surrounding space, this distance comprises less intermediate edges $\Delta z^{\prime}$, making

$$
\Delta z^{\prime}=\Delta z \sqrt{1-v^{2}}
$$

being the expression of the length contraction.

In a lattices-like discrete space, length contraction arises from the same coordination number of all vertices instead of the principle of relativity. In addition, it is based on a high regularity in the presence of anomalies in a hypercubic lattice as discrete space.

\subsection{The Moving Space}

A moving field granule consists of a cube with four moving side planes. All these planes are moving with the same speed $\mathrm{v}$ in the same spatial direction. This caused all the vertices an edges of the cube's interior also moves with speed $\mathrm{v}$ due to the interconnections with the side planes. Like the side planes, the entire space within the moving space will also have time dilation and length contraction as well as a regular space structure.

The consequence of the regular space structure is that each field granule positioned within the moving space has the same internal structure as placed in the non-moving situation, which is the subject of sections 3.3 and 4.2 .

\section{Concluding Remarks}

This article is about the possibility that fields are made up of granules, being three-dimensional cubic-like regions in $\mathrm{R}^{4}$ of finite sizes. There are some indications, to see Chapter 2, that fields in a discrete space-time have such a property.

Consequence of granulation is that the motion of the particle field needs to be split into an inner and an outer part. The inner part moves gradually with a moving space as point-like particles (8), the outer part coupled thereto consists of a series of jumping granules (9) that exhibit wave characteristics.

The dual description of the particle motion results in an expression for the 
discrete Planck's constant (11) in a multidirectional discrete space, the same as obtained in ref. [6].

As shown in Chapter 5, deviations in the regular structure of a hypercubic lattice offer a promising possibility of being the origin of granulations. The attractive thing about space deviations is that it opens up the possibility that all phenomena are the result of structural deviations, i.e. expressions of space itself.

\section{Results}

A discrete space in the form of a hypercubic lattice leads to field granulation.

In the case of granulated fields, the particle motion needs to be split into parts, reminiscent of the particle-wave duality. The particle wave-duality is thus an indication of the granularity of fields besides the existence of the electron radius.

Motion in a static four-dimensional lattice takes place as a space-time corridor in $\mathrm{R}^{4}$ with tilted time axes, to be visualized in a Minkowski diagram-like fashion.

In a hypercubic lattice, the moving corridor is the cause of both movement and relativistic features. The latter arises from regularities in the presence of the anomalies in space structure and from the same coordination number of all vertices.

If the granulation of fields is based on structural anomalies, the multidirectional hypercubic lattice is an ultimate substantival space [10].

With the finding here that motion is relative to space itself, the luminiferous aether discussion of the early $20 \mathrm{e}$ century can be extended with the possibility of a multidirectional hypercubic lattice as the fixed frame of reference.

\section{Conflicts of Interest}

The author declares no conflicts of interest regarding the publication of this paper.

\section{References}

[1] Grünbaum, A. (1967) Modern Science and Zeno's Paradox. Wesleyan University Press, Middletown, Connecticut.

[2] Russell, B. (1926) Our Knowledge of the External World. Allen \& Unwin, London, 179.

[3] Čapek, M. (1961) The Philosophical Impact of Contemporary Physics. Princeton University Press, Princeton, New Jersey, 68-69.

[4] Crisp, T.M. (2007) Presentism, Eternalism and Relativity Physics. In: Craig, W.L. and Smith, Q., Eds., Einstein, Relativity and Absolute Simultaneity, Routledge, London, 262-278.

[5] de Groot, C.T. (2017) Journal of Modern Physics, 8, 1175-1189. https://doi.org/10.4236/jmp.2017.88078

[6] de Groot, C.T. (2020) Journal of Modern Physics, 11, 432-447. https://doi.org/10.4236/jmp.2020.113027

[7] van Bendegem, J.P. (1995) Logique \& Analyse, 38, 127-150.

[8] Grünbaum, A. (1973) Philosophical Problems of Space and Time. 2nd Edition, Rei- 
del, Dordrecht, 632. https://doi.org/10.1007/978-94-010-2622-2

[9] Forrest, P. (1995) Synthese, 103, 327-354. https://doi.org/10.1007/BF01089732

[10] Dasgupta, S. (2015) Philosophy Compass, 10, 601-624.

https://doi.org/10.1111/phc3.12219

\section{Appendix A. The Interconnection of Time Slices}

In the description of an active plane of Figure 5 and Figure 6, there are one or two open edges each $\mathrm{T}$, both at bottom and at the top of the time slices. The vertex with one open edge is connected to a vertex of one of the two adjacent slices, the vertex with two open edges is connected to both adjacent slices. These open edges form the mutual connection of a bundle of time slices. A bundle of $L$ time slices forms a spatial plane, whereby the double open edges uniting the plane and the single open edge causing the plane to have an open edge every $T$.
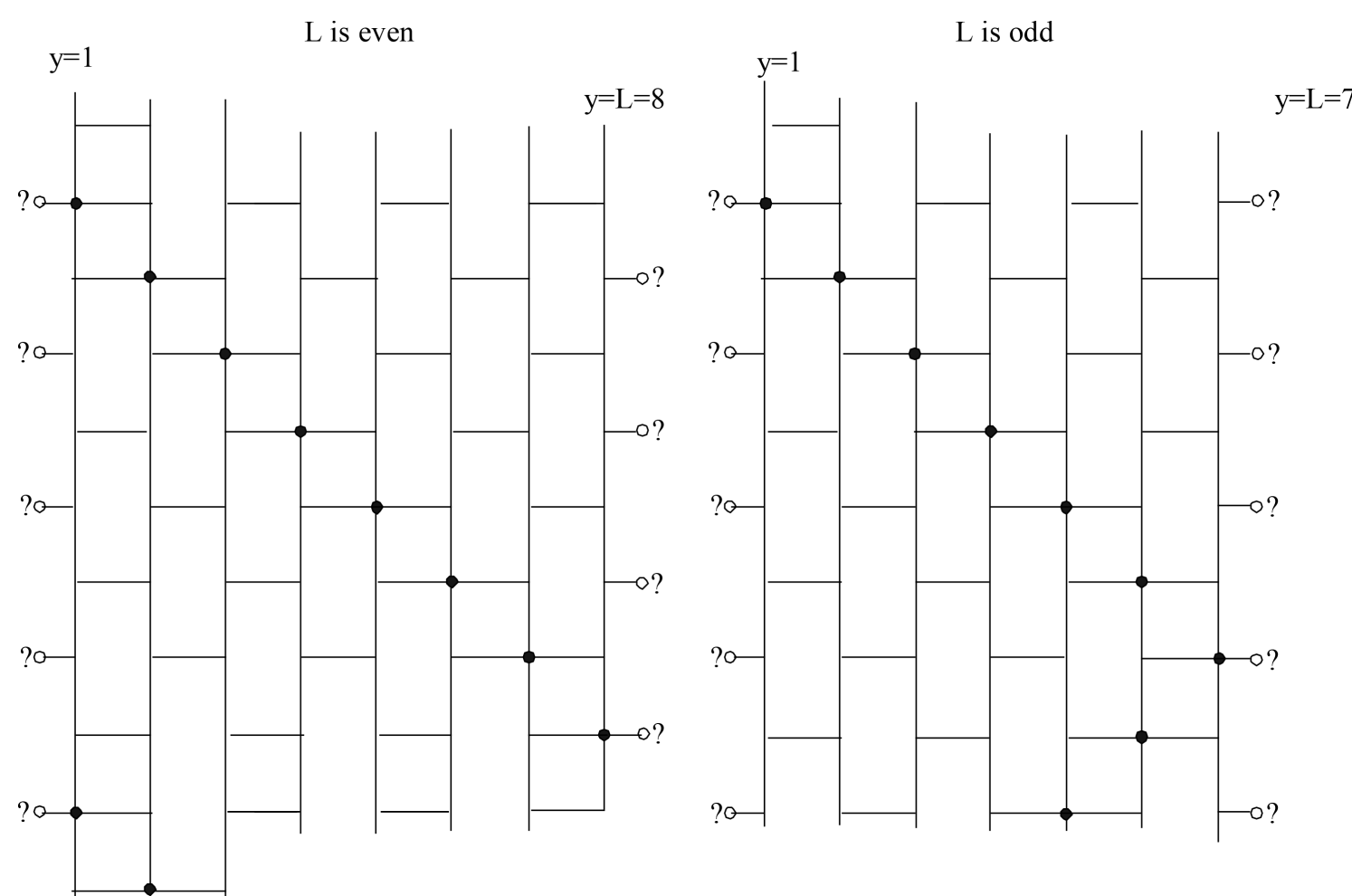

Figure A1. The edges connecting the time slices. Depicted in the $y, t$-plane is the possible organization of the coupling of the time slices via one or two open edges. Due to two open edges there is a regular pattern over the time distance $\mathrm{T}^{2}$ in the $y$, $t$-plane. There are two possible organizations, in the left figure the plane has one open edge on each $T$ at alternating left and right corner vertex, on the right plane there is one open edge on each corner every $2 T$. 